\title{
PAPILIONOIDEA (LEPIDOPTERA: RHOPALOCERA) DE LA SIERRA NEVADA, MÉXICO
}

\author{
Mercedes LUNA-REYES ${ }^{1}$ \& Jorge LLORENTE-BOUSQUETS ${ }^{2}$ \\ ${ }^{1}$ Museo de Zoología, Facultad de Estudios Superiores Zaragoza, \\ Av. Guelatao No. 66. Col. Ejército de Oriente. Iztapalapa. México D. F. 09230 MÉXICO \\ mmluna@puma2.zaragoza.unam.mx \\ ${ }^{2}$ Museo de Zoología, Facultad de Ciencias, UNAM. \\ Apdo Postal 70-399, México D. F. 04510 MÉXICO \\ jlb@hp.fciencias.unam.mx \\ RESUMEN
}

\begin{abstract}
Se realizó un estudio sobre la distribución local, altitudinal y por comunidad vegetacional de las mariposas de la superfamilia Papilionoidea en 37 localidades de las cuatro entidades que comprende la Sierra Nevada. El área se encuentra entre los 1900 y los 3800 msnm y presenta las siguientes comunidades vegetacionales: bosque mesófilo de montaña, de oyamel, Alnus, encino, encino-pino, pino, pino-encino, bosque caducifolio, selva baja caducifolia-bosque mesófilo de montaña, vegetación perturbada y cultivos. En este trabajo se registraron 75 especies, de las cuales 34 son nuevos registros para el área. Se integró una relación actualizada de 85 especies con base en la literatura y en los registros obtenidos en el trabajo de campo. El mayor número de especies correspondió a la familia Nymphalidae. En el análisis espacial de los papilionoideos se encontró que la mayor riqueza y abundancia ocurrió en la localidad de Santa Catarina, en el Estado de México. Se observó que aunque la riqueza específica entre la vertiente occidental y la oriental fue la misma (58 especies), su composición faunística es distinta. Con respecto a la distribución por vegetación, se encontró que en la vertiente oriental las especies de Papilionoidea mostraron los valores más altos y que el bosque mesófilo de montaña y el de encino fueron las comunidades más ricas y abundantes de las 11 reconocidas en el estudio.

Palabras Clave: Distribución altitudinal, local y vegetacional, Lepidoptera, Papilionoidea, parques nacionales, riqueza, Sierra Nevada.
\end{abstract}

\section{ABSTRACT}

The local, altitudinal and vegetational distributions of the superfamily Papilionoidea were studied in 37 localities of the Sierra Nevada. Estimate richness was of 91 species, using species accumulation curves. As a result of this work 75 species were found, 34 of which were new records for the area. An updated species list (85 taxa) was elaborated adding reports of previous works. The Nymphalidae had the greatest number of species. The spatial analysis of the Papilionoidea showed that the highest richness and abundance occurred in the locality of Santa Catarina, México State. In spite of the similarities of the specific richness between occidental and oriental slopes, their faunistic composition were different. The species of the Papilionoidea were greatest in the oriental slope, and the cloud and Quercus forest have the highest value in richness and abundance among the 11 vegetational communities recognized.

Key words: Altitudinal, local and vegetational distributions, Lepidoptera, national parks, Papilionoidea, richness, Sierra Nevada.

\section{INTRODUCCIÓN}

En nuestro país existen más de 300 áreas naturales protegidas (ANP), de las cuales 166 han sido decretadas por el gobierno federal o estatal, el resto fueron establecidas por un instituto de investigación, universidad o propuestas para su protección por 
alguna organización conservacionista no gubernamental (Flores \& Gerez 1994). De las nueve categorías de manejo reconocidas por la Unión Internacional para la Conservación de la Naturaleza (IUCN), en México se utilizan siete que agrupan áreas protegidas decretadas en función de los objetivos que cada una tiene: Reserva Científica, Reserva Natural Estricta; Parques Nacionales; Monumento Natural; Reserva Natural Manejada, Refugios y Santuarios de Vida Silvestre; Paisajes Protegidos, Áreas de Recreación; Reserva de Recursos y Reservas de la Biosfera. Aunque la mayor parte de las áreas protegidas corresponde a Parques Nacionales, la categoría de Reservas de la Biosfera ocupa la mayor extensión territorial y por lo tanto, la mayor superficie total protegida (Flores \& Gerez 1994).

Siete de los 48 parques nacionales que existen en el territorio nacional son compartidos por una o más entidades federativas (Flores \& Gerez 1994), como en el caso de Zoquiapan y Anexas e Izta-Popo, decretados en 1937 y 1948 respectivamente. Considerando la regionalización efectuada por la Comisión Nacional para la Conservación de la Biodiversidad (CONABIO), ambos parques están comprendidos en la Región Prioritaria Terrestre (RTP) 107 denominada como Sierra Nevada (Arriaga et al. 2000).

La Sierra Nevada representa un área de gran importancia desde el punto de vista biogeográfico puesto que ahí convergen las regiones neártica y neotropical, además de que de ella se han citado valores altos de riqueza y endemismos (Arriaga et al. 2000). Presenta un amplio gradiente altitudinal con distintos tipos de vegetación y microhábitats (Arriaga et al. 2000). La mayor parte de los trabajos desarrollados en la Sierra Nevada han sido enfocados al estudio de vertebrados, plantas vasculares y hongos (Arriaga et al. 2000) y muy pocos o ninguno sobre otros taxones. Tal es el caso de las comunidades de las mariposas, para las que se realizó una tesis sobre Papilionoidea en una zona de bosque mesófilo en la localidad de San Rafael en el Estado de México (Barrera \& Romero 1986), que representa la única referencia que existe sobre el estudio formal del grupo en el área.

El análisis de la riqueza faunística muestra que en nuestro país habita alrededor del $10 \%$ de los vertebrados del mundo y que las especies endémicas son numerosas, como en el caso de las aves (10\%) y de más de la mitad de la herpetofauna (Flores 1993, Navarro \& Benítez 1993, Arita \& León 1993). Entre los insectos, el orden Lepidoptera no es la excepción ya que en el territorio nacional se ha registrado alrededor del $12 \%$ de las 146,277 especies de mariposas reconocidas a nivel mundial (Heppner 1991, Luis et al. 2000, 2003); Papilionoidea es una de las más diversas de las 27 superfamilias que constituyen el orden (Llorente et al. 1996, Heppner 1998). Algunas estimaciones sugieren que solo en México habitan alrededor de 1800 especies de papilionoideos (De la Maza 1987, Luis et al. 2003).

El presente trabajo constituye un estudio sobre las mariposas que habitan en la Sierra Nevada cuyo propósito es conocer la composición faunística de los Papilionoidea y describir su abundancia y distribución local, considerando un gradiente altitudinal (1900-3800 m) y el análisis por tipos de vegetación. Este trabajo contribuye al conocimiento de la fauna local de mariposas de montaña, en especial por encima de los $2500 \mathrm{msnm}$, de la cual se sabe muy poco. 


\section{MATERIAL Y MÉTODOS}

Trabajo de campo. Se efectuaron 13 colectas mensuales de cuatro días de duración para obtener el material entomológico, desde junio de 2001 a junio de 2002, período en el que se visitaron 49 localidades ubicadas en un gradiente altitudinal que comprende desde los 1900 a los $3800 \mathrm{~m}$ y en varias comunidades vegetacionales presentes en la zona de estudio (Fig. 1). Los sitios estudiados corresponden a los municipios de Amecameca, Chalco, Ecatzingo, Ixtapaluca, Texcoco y Tlalmanalco en el Estado de México; a Ciudad Nanacamilpa en Tlaxcala; Atlixco, Chiautzingo, Huejotzingo, San Nicolás de los Ranchos, San Salvador El Verde, Tianguismanalco, Tochimilco y Tlahuapan en Puebla, y Tetela del Volcán en Morelos. La información respecto a la altitud y a la vegetación que caracteriza cada uno de los sitios donde fueron registrados papilionoideos se sintetiza en el Cuadro 1.

En cada localidad se realizaron recorridos de 4 horas de duración por al menos dos personas. La colecta de los imagos se logró con ayuda de una red entomológica aérea; buscando entre la vegetación, caminos, veredas y en cualquier lugar en donde se observaron mariposas. Cada ejemplar fue sacrificado y colocado en una bolsa de papel glassine a la que se le anotaron los datos de colecta.

Determinación taxonómica. Se preparó una muestra representativa de todas las especies de acuerdo con las técnicas sugeridas por Howe (1975). Para la determinación taxonómica de los ejemplares se consultaron las obras de Ehrlich y Ehrlich (1961), Emmel (1975), Pyle y Knopf (1981), Scott (1986), De la Maza (1987), DeVries (1987), Smart (1989) y Llorente et al. (1997) y también se tomaron como referencia las colecciones lepidopterológicas de los Museos de Zoología de la FES Zaragoza y de la Facultad de Ciencias, ambos de la UNAM. Todos los ejemplares fueron registrados asignándoles un número consecutivo, agregando los datos de colecta y taxonómicos, y fueron rotulados y depositados en la colección de la FES Zaragoza. En este registro también se incluyó la información de las especies observadas.

Manejo de la información. Se diseñó una base de datos en el programa EXCEL 97 en donde se incluyeron los campos necesarios que contienen la información de colecta, la taxonómica y los datos geográficos (obtenidos con un geoposicionador marca GARMIN mod. $12 \mathrm{XL}$ ) disponibles para cada ejemplar: número del ejemplar, nombre de la localidad, fecha, hora, nombre del recolector, nombre científico de la especie, sexo del individuo, tipo de vegetación, latitud, longitud y altitud. A partir de esta base de datos se generaron tabulaciones y figuras que permitieron hacer el análisis de la riqueza específica de Papilionoidea en la totalidad del área de estudio, del número de especies y de ejemplares por localidad y de su distribución por altitud y por tipo de vegetación. Los resultados obtenidos en este estudio fueron comparados con los registrados en áreas próximas con características similares a la que aquí nos ocupa. 
Luna-Reyes \& Llorente-Bousquets: Papilionoideos de la Sierra Nevada, México

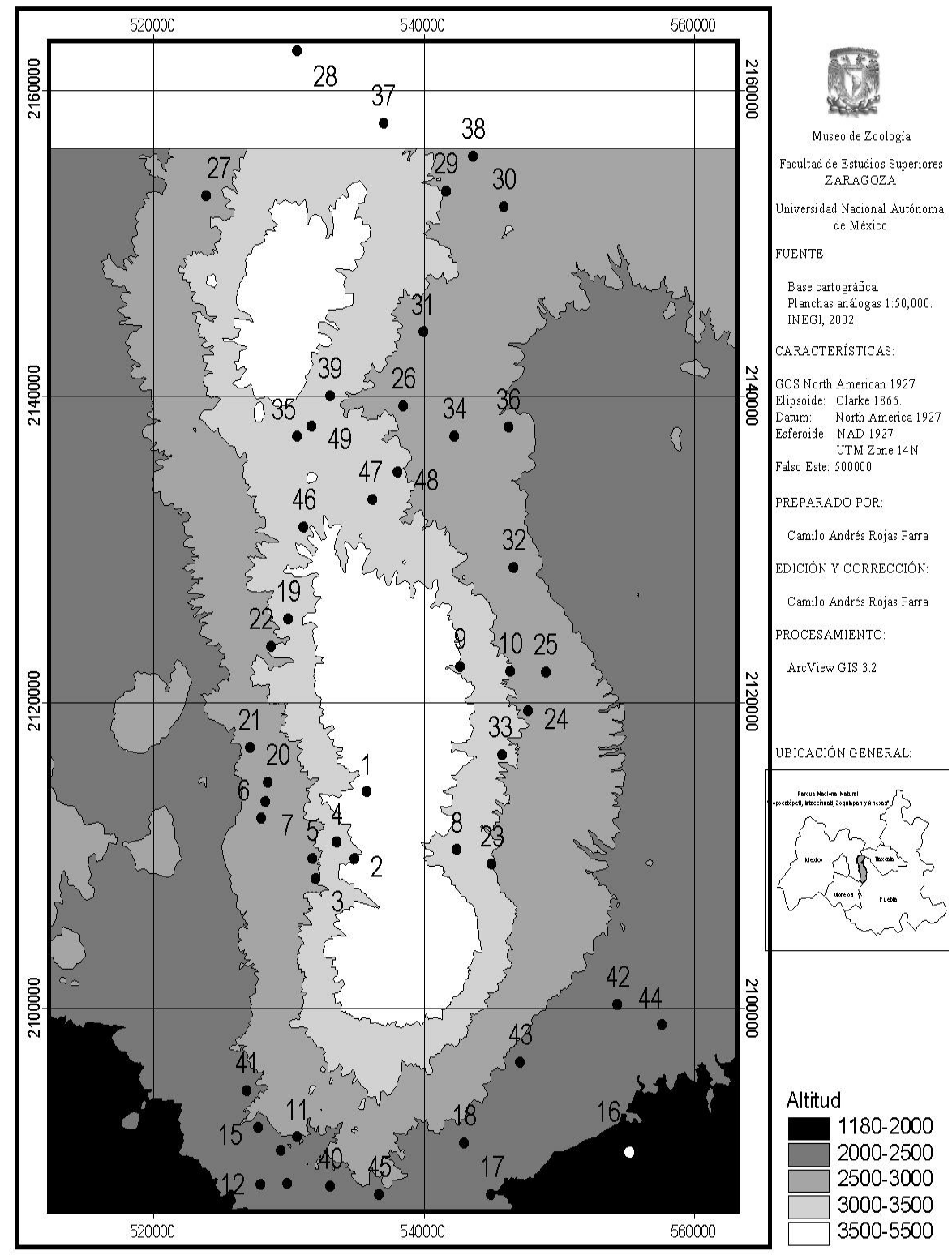

10 10 20 Kilómetros

Figura 1

Ubicación de los sitios de colecta en la Sierra Nevada. Los números corresponden a las localidades descritas en el Cuadro 1. 
Acta Zool. Mex. (n.s.) 20(2) (2004)

Cuadro 1

Localidades estudiadas en la Sierra Nevada. El número de las localidades corresponde al de la figura 1. El asterisco indica los sitios en los que fueron registrados papilionoideos.

\begin{tabular}{|c|c|c|c|c|c|c|c|c|c|c|c|}
\hline No. & - Localidad & Clave & Estado & Lat. $\mathbf{N}$ & Long. 0 & $\begin{array}{l}\text { Altitud } \\
\text { (msnm) }\end{array}$ & Vertiente & Vegetación & Riq & Abund & $\begin{array}{c}\text { E. de C. } \\
\text { horas }\end{array}$ \\
\hline$\overline{1}$ & Altzomoni & AT & México & $19^{\circ} 07^{\prime} 14.7^{\prime \prime}$ & $98^{\circ} 39^{\prime} 34.05^{\prime \prime}$ & 3800 & OCC & $\mathrm{ZA}$ & & & 20 \\
\hline 2 & Pinar & PR & México & $19^{\circ} 04^{\prime} 53.2^{\prime \prime}$ & $98^{\circ} 40^{\prime} 05.2^{\prime \prime}$ & 3600 & OCC & $\mathrm{BP}$ & & & 20 \\
\hline 3 & *La Tijera & $\mathrm{TJ}$ & México & $19^{\circ} 04^{\prime} 10.6^{\prime \prime}$ & $98^{\circ} 41^{\prime} 43.4^{\prime \prime}$ & 3100 & OCC & BCA & 1 & 2 & 20 \\
\hline 4 & La Cañada & CÑ & México & $19^{\circ} 05^{\prime} 29.1^{\prime \prime}$ & $98^{\circ} 40^{\prime} 50.3^{\prime \prime}$ & 3300 & OCC & BY & & & 20 \\
\hline 5 & *Viveros & VV & México & $19^{\circ} 04^{\prime} 53.9^{\prime \prime}$ & $98^{\circ} 41^{\prime} 52.9^{\prime \prime}$ & 2900 & OCC & BY & 3 & 3 & 20 \\
\hline 6 & *Tomacocos & TM & México & $19^{\circ} 06^{\prime} 18.8^{\prime \prime}$ & $98^{\circ} 44^{\prime} 02.1^{\prime \prime}$ & 2500 & OCC & BPE & 8 & 11 & 20 \\
\hline 7 & Alcalica & $A L$ & México & $19^{\circ} 06^{\prime} 54.5^{\prime \prime}$ & $98^{\circ} 43^{\prime} 50.9^{\prime \prime}$ & 2700 & $\mathrm{OCC}$ & $\mathrm{BP}$ & & & 20 \\
\hline 8 & *Buenavista & BV & Puebla & $19^{\circ} 05^{\prime} 11.2^{\prime \prime}$ & $98^{\circ} 35^{\prime} 46.2^{\prime \prime}$ & 3300 & OTE & $\mathrm{BAl}$ & 3 & 5 & 20 \\
\hline 9 & *San Juan Tetla & JT & Puebla & $19^{\circ} 11^{\prime} 40.6^{\prime \prime}$ & $98^{\circ} 35^{\prime} 37.8^{\prime \prime}$ & 3400 & OTE & BPE & 8 & 26 & 20 \\
\hline 10 & ${ }^{*}$ Camino a San Juan & CJ & Puebla & $19^{\circ} 11^{\prime} 31.3^{\prime \prime}$ & $98^{\circ} 33^{\prime} 29.6^{\prime \prime}$ & 2800 & OTE & BPE & 5 & 19 & 20 \\
\hline 11 & Canistitla & $\mathrm{CN}$ & Morelos & $18^{\circ} 55^{\prime} 01^{\prime \prime}$ & $98^{\circ} 42^{\prime} 32.3^{\prime \prime}$ & 2500 & OCC & $\mathrm{BE}$ & & & 20 \\
\hline 12 & *Tetela & $\mathrm{TT}$ & Morelos & $18^{\circ} 53^{\prime} 20.5^{\prime \prime}$ & $98^{\circ} 44^{\prime} 04.1^{\prime \prime}$ & 2200 & OCC & BCA & 18 & 34 & 20 \\
\hline 13 & Cerro de Tetela & CT & Morelos & $18^{\circ} 54^{\prime} 33.3^{\prime \prime}$ & $98^{\circ} 43^{\prime} 13^{\prime \prime}$ & 2400 & OCC & $\mathrm{BE}$ & & & 20 \\
\hline 14 & ${ }^{*}$ Camino a Hueyapan & $\mathrm{CH}$ & Morelos & $18^{\circ} 53^{\prime} 21.3^{\prime \prime}$ & $98^{\circ} 42^{\prime} 57.6^{\prime \prime}$ & 2200 & OCC & C & 2 & 11 & 20 \\
\hline 15 & ${ }^{*}$ Ocoxaltepec & OX & Morelos & $18^{\circ} 55^{\prime} 22.4^{\prime \prime}$ & $98^{\circ} 44^{\prime} 11^{\prime \prime}$ & 2400 & OCC & BPE & 6 & 6 & 20 \\
\hline 16 & *San Alejo & AJ & Puebla & $18^{\circ} 54^{\prime} 25.8^{\prime \prime}$ & $98^{\circ} 28^{\prime} 31.6^{\prime \prime}$ & 1900 & OTE & C & 17 & 30 & 4 \\
\hline 17 & *Tochimilco & $\mathrm{TH}$ & Puebla & $18^{\circ} 52^{\prime} 58.7^{\prime \prime}$ & $98^{\circ} 34^{\prime} 21.6^{\prime \prime}$ & 2000 & OTE & SBC-BMM & 17 & 29 & 4 \\
\hline 18 & Santa Magdalena MG & Puebla & $18^{\circ} 54^{\prime} 47$ & $.7^{\prime \prime} \quad 98^{\circ}$ & $35^{\prime} 29^{\prime \prime} \quad 2300$ & 0 OTE & $\mathrm{BCA}$ & & & 4 & \\
\hline 19 & *Ejido Tlalmanalco & TL & México & $19^{\circ} 13^{\prime} 23.6^{\prime \prime}$ & $98^{\circ} 42^{\prime} 52^{\prime \prime}$ & 3100 & OCC & $\mathrm{BCA}$ & 4 & 4 & 8 \\
\hline 20 & ${ }^{*}$ El Salto & SL & México & $19^{\circ} 07^{\prime} 35.9^{\prime \prime}$ & $98^{\circ} 43^{\prime} 44.1^{\prime \prime}$ & 2700 & OCC & BPE & 19 & 66 & 8 \\
\hline 21 & Santiago & SG & México & $19^{\circ} 08^{\prime} 49.9^{\prime \prime}$ & $98^{\circ} 44^{\prime} 29.8^{\prime \prime}$ & 2600 & OCC & BE & & & 8 \\
\hline 22 & *San Rafael & SR & México & $19^{\circ} 12^{\prime} 23.8^{\prime \prime}$ & $98^{\circ} 43^{\prime} 35^{\prime \prime}$ & 3100 & $\mathrm{OCC}$ & $\mathrm{BP}$ & 1 & 3 & 8 \\
\hline 23 & *Nicolás & $\mathrm{NC}$ & Puebla & $19^{\circ} 04^{\prime} 40.6^{\prime \prime}$ & $98^{\circ} 34^{\prime} 18.6^{\prime \prime}$ & 3100 & OTE & BPE & 2 & 6 & 8 \\
\hline 24 & ${ }^{*}$ Tlatenco & TN & Puebla & $19^{\circ} 10^{\prime} 07.0^{\prime \prime}$ & $98^{\circ} 32^{\prime} 44.8^{\prime \prime}$ & 3000 & OTE & $\mathrm{BP}$ & 10 & 23 & 8 \\
\hline 25 & ${ }^{*}$ Atzompa & $A Z$ & Puebla & $19^{\circ} 11^{\prime} 28.3^{\prime \prime}$ & $98^{\circ} 32^{\prime} 22.6^{\prime \prime}$ & 2700 & OTE & BE & 7 & 51 & 8 \\
\hline 26 & *Santa Rita & RT & Puebla & $19^{\circ} 20^{\prime} 55.1^{\prime \prime}$ & $98^{\circ} 37^{\prime} 59.3^{\prime \prime}$ & 2900 & OTE & BPE & 1 & 1 & 8 \\
\hline 27 & *Santa Catarina & SC & México & $19^{\circ} 28^{\prime} 22.7^{\prime \prime}$ & $98^{\circ} 46^{\prime} 18^{\prime \prime}$ & 2800 & OCC & $\mathrm{BE}$ & 25 & 88 & 8 \\
\hline 28 & Límite & LM & México & $19^{\circ} 33^{\prime} 30.7^{\prime \prime}$ & $98^{\circ} 42^{\prime} 27.4^{\prime \prime}$ & 2900 & OCC & PAZ & & & 8 \\
\hline 29 & ${ }^{*}$ El Sifón & SF & Tlaxcala & $19^{\circ} 28^{\prime} 32^{\prime \prime}$ & $98^{\circ} 36^{\prime} 09.7^{\prime \prime}$ & 3000 & OTE & BEP & 15 & 55 & 8 \\
\hline 30 & San Felipe Hidalgo & $\mathrm{FH}$ & Tlaxcala & $19^{\circ} 27^{\prime} 57.5^{\prime \prime}$ & $98^{\circ} 33^{\prime} 43.8^{\prime \prime}$ & 2800 & OTE & $\mathrm{BCA}$ & & & 8 \\
\hline 31 & ${ }^{*}$ Cuauhtémoc & $\mathrm{CU}$ & Puebla & $19^{\circ} 23^{\prime} 32.3^{\prime \prime}$ & $98^{\circ} 37^{\prime} 09^{\prime \prime}$ & 2800 & OTE & $\mathrm{BP}$ & 1 & 1 & 8 \\
\hline 32 & *San Andrés & AN & Puebla & $19^{\circ} 15^{\prime} 10.4^{\prime \prime}$ & $98^{\circ} 33^{\prime} 20.7^{\prime \prime}$ & 2600 & OTE & BPE & 4 & 10 & 8 \\
\hline 33 & ${ }^{*}$ Atexcac & $A X$ & Puebla & $19^{\circ} 08^{\prime} 32.9^{\prime \prime}$ & $98^{\circ} 33^{\prime} 50.1^{\prime \prime}$ & 3100 & OTE & BPE & 8 & 21 & 8 \\
\hline 34 & *Avila Camacho & $A C$ & México & $19^{\circ} 19^{\prime} 49.9^{\prime \prime}$ & $98^{\circ} 35^{\prime} 50.1^{\prime \prime}$ & 2800 & OCC & $\mathrm{BE}$ & 6 & 7 & 8 \\
\hline 35 & *Llano Grande & LL & México & $19^{\circ} 19^{\prime} 50.4^{\prime \prime}$ & $98^{\circ} 42^{\prime} 30.3^{\prime \prime}$ & 3300 & OCC & $\mathrm{BCA}$ & 5 & 5 & 8 \\
\hline 36 & Apapaxco & AP & Tlaxcala & $19^{\circ} 20^{\prime} 09.2^{\prime \prime}$ & $98^{\circ} 33^{\prime} 33.3^{\prime \prime}$ & 2600 & OTE & $\mathrm{BCu}$ & & & 8 \\
\hline 37 & *La Soledad & SO & Tlaxcala & $19^{\circ} 30^{\prime} 56.2^{\prime \prime}$ & $98^{\circ} 38^{\prime} 48.9^{\prime \prime}$ & 3000 & OTE & $\mathrm{BP}$ & 6 & 13 & 8 \\
\hline 38 & *La Vaquería & VQ & Tlaxcala & $19^{\circ} 29^{\prime} 46.7^{\prime \prime}$ & $98^{\circ} 35^{\prime} 0.9^{\prime \prime}$ & 2800 & OTE & BP & 2 & 3 & 8 \\
\hline 39 & *Río Frío & RF & México & $19^{\circ} 21^{\prime} 18^{\prime \prime}$ & $98^{\circ} 41^{\prime} 05.8^{\prime \prime}$ & 3100 & OTE & BPE & 8 & 8 & 8 \\
\hline 40 & *Hueyapan & HY & Morelos & $18^{\circ} 53^{\prime} 16.5^{\prime \prime}$ & $98^{\circ} 41^{\prime} 07.3^{\prime \prime}$ & 2300 & OCC & ZP & 13 & 14 & 8 \\
\hline 41 & ${ }^{*}$ Ecatzingo & $\mathrm{EC}$ & México & $18^{\circ} 56^{\prime} 39.9^{\prime \prime}$ & $98^{\circ} 44^{\prime} 39.1^{\prime \prime}$ & 2400 & OCC & BMM & 24 & 55 & 8 \\
\hline 42 & *Atlimeyaya & AY & Puebla & $18^{\circ} 59^{\prime} 40.6^{\prime \prime}$ & $98^{\circ} 29^{\prime} 00^{\prime \prime}$ & 2200 & OTE & BPE & 8 & 10 & 8 \\
\hline 43 & ${ }^{*}$ San Pedro & BJ & Puebla & $18^{\circ} 57^{\prime} 38.9^{\prime \prime}$ & $98^{\circ} 33^{\prime} 6.5^{\prime \prime}$ & 2400 & OTE & BPE & 7 & 20 & 8 \\
\hline 44 & *Tianguismanalco & TG & Puebla & $18^{\circ} 58^{\prime} 57.2^{\prime \prime}$ & $98^{\circ} 27^{\prime} 7.4^{\prime \prime}$ & 2100 & OTE & ZP & 2 & 2 & 8 \\
\hline 45 & *Santa Cruz & $\mathrm{CZ}$ & Puebla & $18^{\circ} 52^{\prime} 59.2^{\prime \prime}$ & $98^{\circ} 39^{\prime} 4.4^{\prime \prime}$ & 2400 & OTE & $\mathrm{BE}$ & 16 & 39 & 8 \\
\hline 46 & Cuautlalpan & $\mathrm{CP}$ & México & $19^{\circ} 16^{\prime} 37.7^{\prime \prime}$ & $98^{\circ} 42^{\prime} 12.9^{\prime \prime}$ & 3300 & OCC & $\mathrm{BCA}$ & & & 8 \\
\hline 47 & *Rancho Los Venados & LV & México & $19^{\circ} 17^{\prime} 37.1^{\prime \prime}$ & $98^{\circ} 39^{\prime} 18.7^{\prime \prime}$ & 3100 & OTE & $\mathrm{BP}$ & 6 & 8 & 8 \\
\hline 48 & ${ }^{*}$ Valle Espinoso & VE & México & $19^{\circ} 18^{\prime} 34.1 "$ & $98^{\circ} 38^{\prime} 13.6^{\prime \prime}$ & 3000 & OTE & BP & 2 & 2 & 8 \\
\hline 49 & ${ }^{*} \mathrm{Km} 40$ & $\mathrm{KM}$ & México & $19^{\circ} 20^{\prime} 13.2^{\prime \prime}$ & $98^{\circ} 41^{\prime} 52.8^{\prime \prime}$ & 3200 & OTE & BEP & 2 & 2 & 8 \\
\hline
\end{tabular}

BCA: bosque caducifolio (mixto de encino y otras hojosas); BAl: bosque caducifolio (mixto de encino y otras hojosas, con dominancia de Alnus); BCu: bosque cultivado (eucalipto, pino, casuarina); BE: bosque de encino; BEP: bosque de encino-pino; BP: bosque de pino; BPE: bosque de pino-encino; BMM: bosque mesófilo de montaña; BY: bosque de oyamel; C: cultivos (maíz, frijol, cebada, pera, manzana, ciruela); PAZ: pastizal; SBC-BMM: ecotono entre selva baja caducifolia y BMM; ZA: zacatonal alpino; ZP: zona perturbada (vegetación riparia). INEGI, 1988. OCC: occidental; OTE: oriental; Riq: riqueza; Abund: abundancia; E. de C.: esfuerzo de captura 
Luna-Reyes \& Llorente-Bousquets: Papilionoideos de la Sierra Nevada, México

Esfuerzo de captura. Se estimó la representatividad del número de especies reconocidas en este trabajo mediante el análisis de los valores de la curva de acumulación de especies observadas y la aplicación del índice de $\mathrm{ChaO}_{2}$ (Colwell \& Coddington 1994) que requiere de los datos de presencia-ausencia de las especies en las distintas localidades de colecta que comprende el área de estudio. El índice se describe a continuación:

donde: $S_{\text {obs }}=$ número de especies observadas

$$
\mathrm{ChaO}_{2}=\mathrm{S}_{\text {obs }}+\left(\mathrm{L}^{2} / 2 \mathrm{M}\right)
$$

$\mathrm{L}=$ número de especies representadas en el inventario de una localidad $\mathrm{M}=$ número de especies representadas en el inventario en dos localidades

\section{RESULTADOS}

Lista de especies. Con base en la determinación taxonómica de los 693 ejemplares registrados (capturados y observados) se integró una relación de 75 especies distribuidas en 56 géneros y 15 subfamilias de cuatro familias propuestas para Papilionoidea: Papilionidae, Pieridae, Nymphalidae y Lycaenidae (sensu Kristensen, 1975).

La lista que se presenta a continuación sigue un ordenamiento filogenético aproximado de acuerdo con Scott (1985) y la nomenclatura más actualizada para el grupo (Llorente et al. datos no publicados). Se indican con un círculo las especies que solo fueron observadas y con un asterisco las que son citadas por primera vez para la Sierra Nevada.

\section{PAPILIONIDAE}

Papilioninae

${ }^{*}$ Battus philenor philenor (Linnaeus, 1771)

Parides photinus photinus (Doubleday, 1844)

*Priamides pharnaces (Doubleday, 1846)

Papilio polyxenes asterius Cramer, 1782

Pterourus multicaudatus (Kirby, 1884)

Pyrrhosticta garamas garamas (Geyer, [1829])

\section{PIERIDAE}

Dismorphiinae

${ }^{*}$ Enantia mazai diazi Llorente, 1984

\section{Coliadinae}

Colias eurytheme Boisduval, 1852

Zerene cesonia cesonia (Stoll, 1791)

Anteos clorinde nivifera (Frühstorfer, 1907)

Anteos maerula lacordairei (Boisduval, 1836)

Phoebis agarithe agarithe (Boisduval, 1836)

${ }^{*}$ Phoebis neocypris virgo (Butler, 1870)

Phoebis philea philea (Linnaeus, 1763)

*Phoebis sennae marcellina (Cramer, 1777) 
Abaeis nicippe (Cramer, 1780)

Pyrisitia nise nelphe (R. Felder, 1869)

*Eurema boisduvaliana (C. Felder \& R. Felder, 1865)

Eurema daira (Godart, 1819)

Eurema mexicana mexicana (Boisduval, 1836)

Eurema salome jamapa (Reakirt, 1866)

Nathalis iole Boisduval, 1836

\section{Pierinae}

${ }^{*}$ Hesperocharis costaricensis pasion (Reakirt, [1867])

Hesperocharis graphites avivolans (Butler, 1865)

Catasticta nimbice nimbice (Boisduval, 1836)

Catasticta teutila teutila (Doubleday, 1847)

Pontia protodice (Boisduval \& LeConte, 1829)

Leptophobia aripa elodia (Boisduval, 1836)

-Ascia monuste monuste (Linnaeus, 1764)

\section{NYMPHALIDAE}

\section{Heliconiinae}

${ }^{*}$ Dione juno huascuma (Reakirt, 1866)

Dione moneta poeyii Butler, 1873

-Agraulis vanillae incarnata (Riley, 1926)

Heliconius charitonia vazquezae Comstock \& F. M. Brown, 1950

*Euptoieta claudia daunius (Herbst, 1798)

${ }^{*}$ Euptoieta hegesia hoffmanni Comstock, 1944

\section{Nymphalinae}

*Vanessa atalanta rubria (Frühstorfer, 1909)

Cynthia annabella (Field, 1971)

Cynthia cardui (Linnaeus, 1758)

Cynthia virginiensis (Drury, 1773)

Nymphalis antiopa antiopa (Linnaeus, 1758)

Polygonia haroldii (Dewitz, 1877)

-*Polygonia sp.

Siproeta stelenes biplagiata (Frühstorfer, 1907)

Junonia coenia Hübner, [1822]

*Anemeca ehrenbergii (Geyer, [1833])

${ }^{*}$ Chlosyne lacinia lacinia (Geyer, 1837)

* Thessalia cyneas cyneas (Godman \& Salvin, 1878)

*Texola elada elada (Hewitson, 1868)

*Phyciodes pictus pallescens (R. Felder, 1869)

*Anthanassa alexon alexon (Godman \& Salvin, 1889)

Anthanassa texana texana (W.H. Edwards, 1863)

\section{Limenitidinae}

${ }^{*}$ Cyclogramma bacchis (Doubleday, [1849])

\section{Apaturinae}

*Doxocopa laure acca (C. Felder \& R. Felder, 1867)

\section{Morphinae}

- Pessonia polyphemus polyphemus Westwood, 1851

\section{Satyrinae}

${ }^{*}$ Cyllopsis pyracmon pyracmon (Butler, 1867)

Paramacera xicaque xicaque (Reakirt, [1867])

*Pindis squamistriga R. Felder, 1869 
Luna-Reyes \& Llorente-Bousquets: Papilionoideos de la Sierra Nevada, México

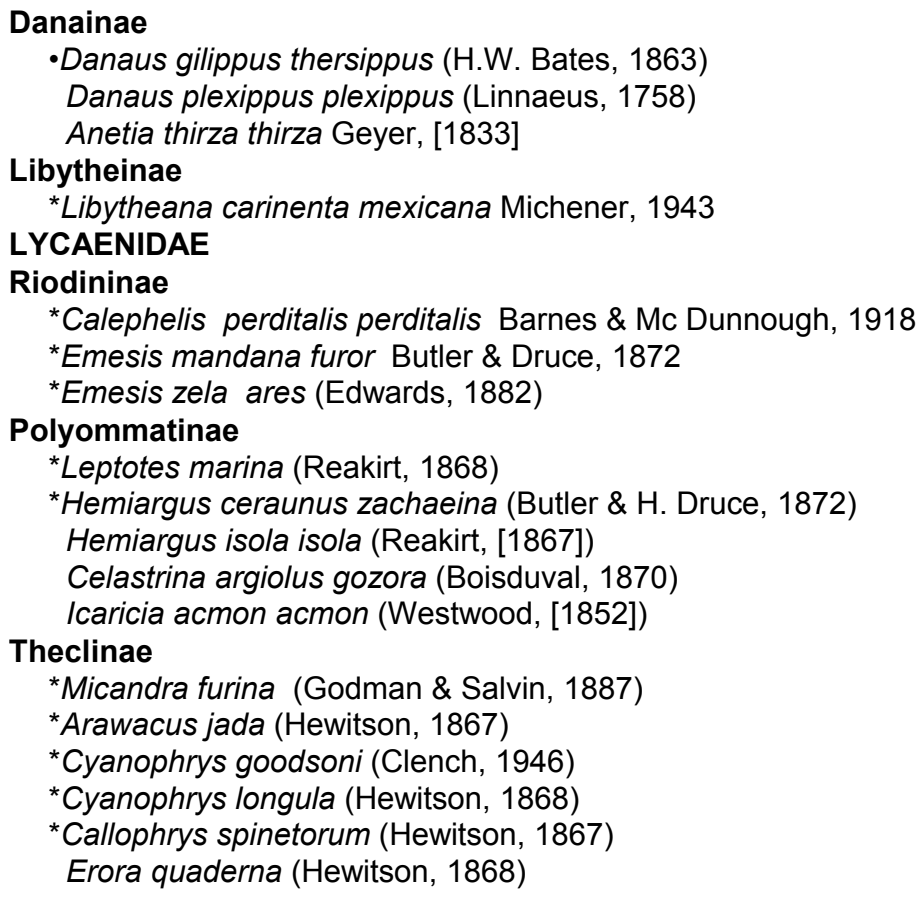

Riqueza. El número de especies encontrado en este estudio (75) fue mayor al obtenido en otros trabajos desarrollados también en zonas montanas. En el Cuadro 2 se sintetizan los resultados de la riqueza de la Sierra Nevada, de la Sierra de Nanchititla (Barrera \& Díaz 1977) y San Rafael (Barrera \& Romero 1986) en el Estado de México, Los Dínamos (Luis y Llorente, 1990) en el D. F. y El Chico (Bizuet et al. 2001) en el Estado de Hidalgo.

En el Cuadro 2 se aprecia además que se encontró la misma proporción de especies por familia que la detectada en estos trabajos: la mayor parte correspondió a Nymphalidae (32), después a Pieridae (23), Lycaenidae (14) y Papilionidae a la cual corresponde el menor número de especies (6), como se ilustra en la figura 2. Aunque coincide la distribución de especies por familia, los valores encontrados en la Sierra Nevada son superiores a los de las otras localidades equivalentes.

Abundancia. El número de registros en la Sierra Nevada fue comparativamente más bajo que el referido para las localidades de San Rafael, Los Dínamos y El Chico (no se conocen los datos de Sierra de Nanchititla). Como se observa en el cuadro 2, la abundancia de Papilionoidea en el área de estudio constituye prácticamente la mitad (693 registros) de la encontrada en la localidad más pobre representada por San Rafael (1412).

Con respecto a la evaluación por familia, Pieridae fue la más abundante con 302 registros, seguida por Nymphalidae (213), Lycaenidae (114) y Papilionidae (64); estos resultados son similares a los registrados en San Rafael y El Chico (Fig. 2, Cuadro 2). 


\section{ESPECIES}

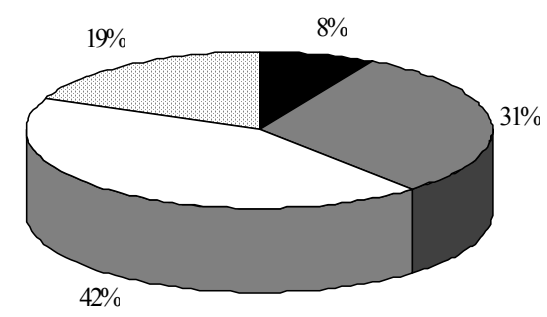

693 EJEMPLARES

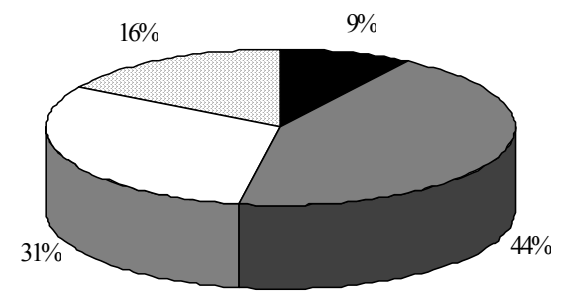

口 Pap $\square$ Pie $\square$ Nym $\square$ Lyc

Figura 2

Riqueza y abundancia de Papilionoidea en la Sierra Nevada. Pap: Papilionidae; Pie: Pieridae; Nym: Nymphalidae; Lyc: Lycaenidae

\section{Cuadro 2}

Comparativo de la riqueza y abundancia de Papilionoidea de la Sierra Nevada con otras localidades

\begin{tabular}{|c|c|c|c|c|c|}
\hline Localidad/Familia & Pap & Pie & Nym & Lyc & Total \\
\hline $\begin{array}{l}\text { Nanchititla, Edo. de Méx. } \\
\text { Barrera y Díaz, } 1977\end{array}$ & 2 & 8 & 18 & 4 & 32 \\
\hline $\begin{array}{l}\text { San Rafael, Edo. de Méx. } \\
\text { Barrera y Romero, } 1986\end{array}$ & $2(38)$ & $18(773)$ & $18(397)$ & $7(38)$ & $45(1412)$ \\
\hline $\begin{array}{l}\text { Los Dínamos, D. F. } \\
\text { Luis y Lorente, } 1990\end{array}$ & $4(29)$ & $18(908)$ & $29(2091)$ & $14(1093)$ & $65(4121)$ \\
\hline $\begin{array}{l}\text { El Chico, Hgo. } \\
\text { Bizuet et al., } 2001\end{array}$ & $5(31)$ & 17 (935) & $32(817)$ & 15 (153) & 69 (1936) \\
\hline $\begin{array}{l}\text { Sierra Nevada } \\
\text { Este estudio }\end{array}$ & $6(64)$ & $23(302)$ & $32(213)$ & $14(114)$ & $75(693)$ \\
\hline
\end{tabular}


En el análisis por especie, se encontró que algunas fueron abundantes, mientras que la mayoría fue representada por pocos ejemplares (Fig. 3).

Del total de especies, Catasticta teutila teutila fue la más abundante; Leptophobia aripa elodia, Paramacera xicaque xicaque, C. nimbice nimbice y Hemiargus isola isola tuvieron más de 40 registros (53, 49, 47 y 41 respectivamente), 11 especies entre 10 y 40 y más del $75 \%$ tuvo menos de 10 ejemplares, gran parte de las cuales solo fue registrada una vez. En el cuadro 3 únicamente se muestra la información de las especies más abundantes y de mayor distribución dentro del área de estudio.

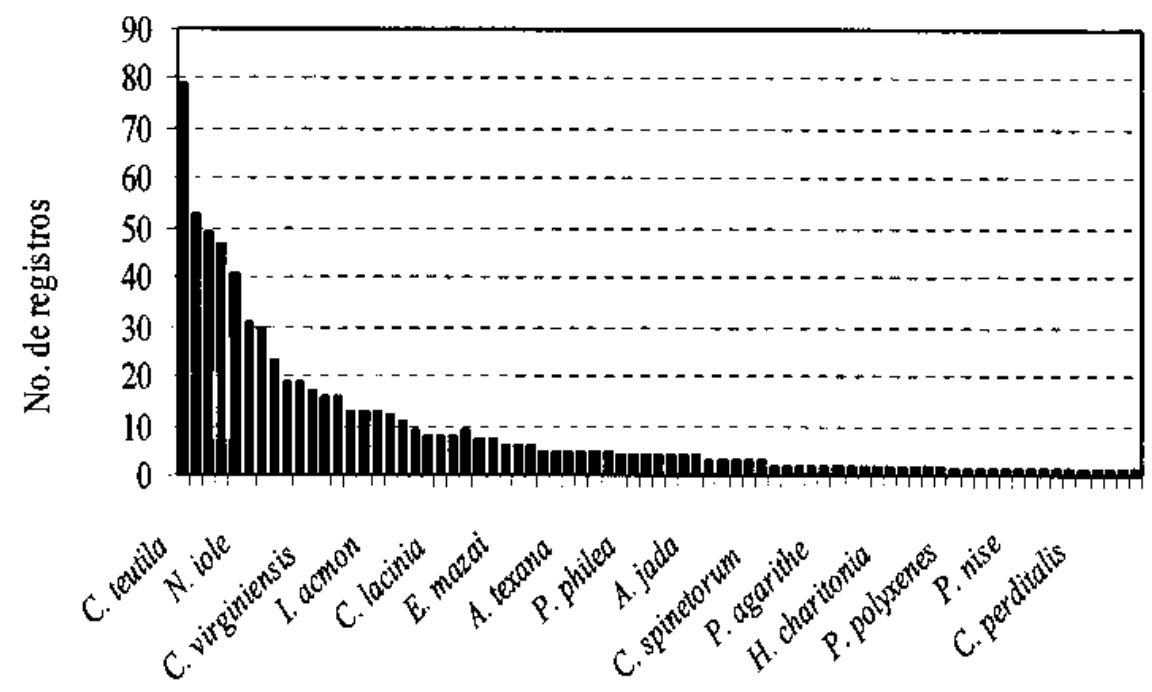

Figura 3

Abundancia de papilionoideos por especie.

Esfuerzo de captura. De acuerdo con el análisis del esfuerzo de colecta efectuado (Colwell \& Coddington 1994), el número estimado de especies para la Sierra Nevada es 91 . En la figura 4 se muestran las curvas de acumulación de especies observadas y la obtenida mediante el modelo de $\mathrm{ChaO}_{2}$; los sitios se presentan en el orden en que fueron estudiados (ver siglas en el Cuadro 2).

Distribución por localidad. Treinta y siete localidades de las 49 visitadas fueron consideradas en el análisis de la distribución espacial de los Papilionoidea del área (Cuadro 2). Algunas condiciones desfavorables para las mariposas como bajas temperaturas, lluvia, neblina, combinaciones entre éstas o las condiciones de la vegetación, impidieron la observación de los imagos en los sitios restantes.

Las localidades que presentaron mayor riqueza fueron Santa Catarina (25 especies) y Ecatzingo (24 especies), ambas ubicadas en el Estado de México, pero en la mayoría de los sitios se detectó una riqueza menor a 10 especies y en algunos como en La Tijera (TJ), San Rafael (SR), Santa Rita (RT) y Cuauhtémoc (CU) solo se capturaron ejemplares de una especie (Cuadro 1). 
Cuadro 3

Distribución por abundancia y número de localidades

\begin{tabular}{lcc}
\hline \multicolumn{1}{c}{ Especie } & No. de registros & No. de localidades \\
\hline Catasticta teutila teutila & 79 & 8 \\
Leptophobia aripa elodia & 53 & 19 \\
Paramacera xicaque xicaque & 49 & 13 \\
Catasticta nimbice nimbice & 47 & 9 \\
Hemiargus isola isola & 41 & 9 \\
Nathalis iole & 31 & 11 \\
Celastrina argiolus gozora & 30 & 10 \\
Battus philenor philenor & 23 & 6 \\
Pterourus multicaudatus & 19 & 12 \\
Cynthia virginiensis & 17 & 6 \\
Zerene cesonia cesonia & 16 & 12 \\
Cynthia cardui & 16 & 8 \\
Pyrrhosticta garamas garamas & 13 & 8 \\
Dione moneta poeyii & 13 & 7 \\
Eurema salome jamapa & 12 & 9 \\
Anteos clorinde nivifera & 11 & 8 \\
Danaus plexippus plexippus & 9 & 5 \\
Eurema mexicana mexicana & 8 & 6 \\
Phoebis sennae marcellina & 7 & 6 \\
Danaus gilippus thersippus & 6 & 5 \\
Eurema daira & 5 & 5 \\
\hline
\end{tabular}

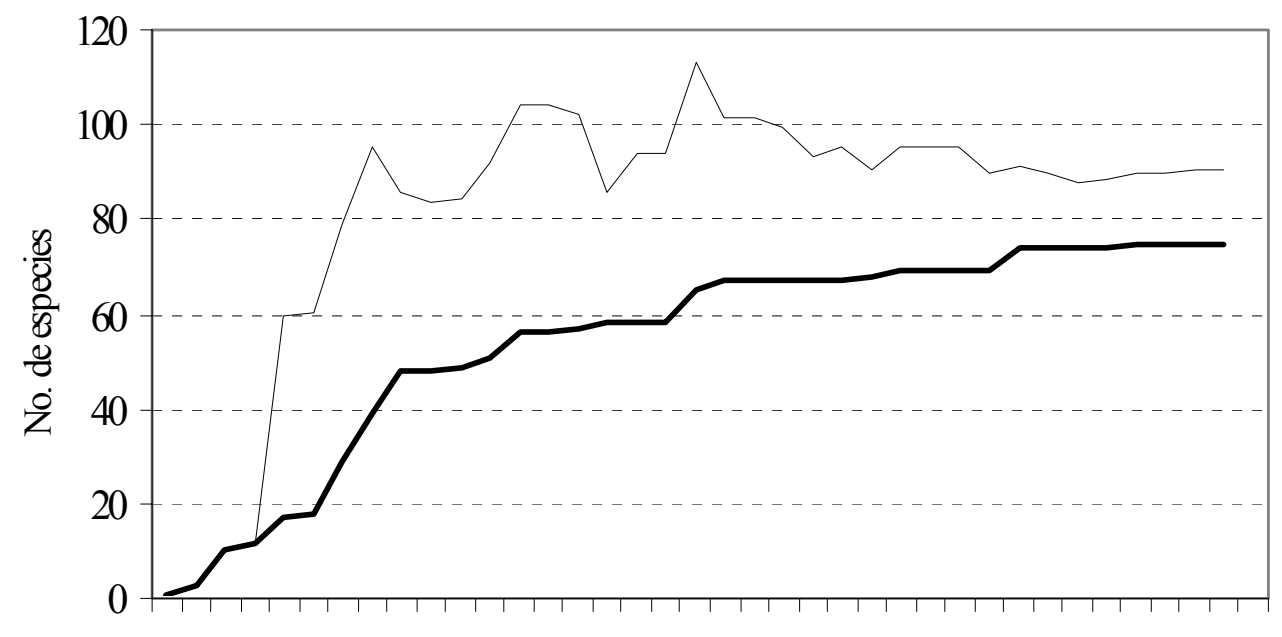

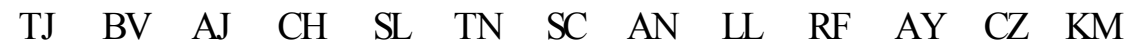

spp ACUM C Chao2

Figura 4

Curva de acumulación de especies. 
Además de ser la localidad más rica, Santa Catarina también presentó el valor más alto de abundancia (88 registros). El Salto (SL), Ecatzingo (EC), El Sifón (SF) y Atzompa (AZ) tuvieron más de 50 registros; solo estas cinco localidades contienen en su conjunto más del $45 \%$ del total registrado. Sin embargo, en la mitad de los sitios hubo diez o menos registros, como en el caso de Santa Rita (RT) y Cuauhtémoc (CU) en los que se capturó un ejemplar solamente. En la figura 5 se expresan los resultados de riqueza y abundancia para cada sitio; las claves de las localidades corresponden a las del Cuadro 1.

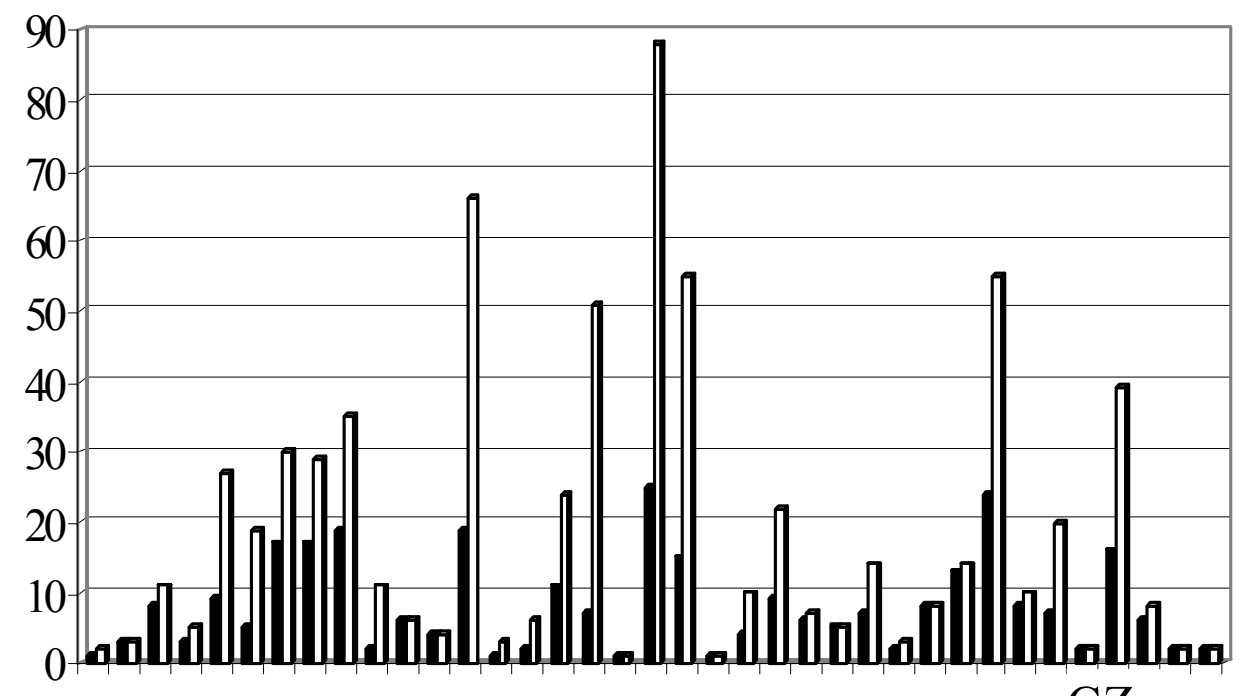

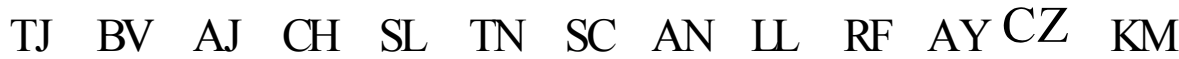

\section{- Riqueza $\square$ Abundancia}

Figura 5

Distribución de Papilionoidea por localidad. Las siglas corresponden a las del Cuadro 1.

En cuanto a la distribución de especies por localidad, en la mitad de los sitios estudiados se encontró al piérido Leptophobia aripa elodia. Sin embargo, más del $70 \%$ de las especies se observó en menos de cinco sitios y un tercio del total se observó solo en una localidad. En el Cuadro 3 se presentan aquellas que estuvieron presentes en cinco o más localidades. A pesar de que Catasticta teutila teutila tuvo la mayor abundancia de Papilionoidea, solo se encontró en ocho sitios, con la mayor concentración de registros en las localidades de Atzompa (30), Santa Catarina (23) y el Salto (19). Otra de las especies abundantes, Paramacera xicaque xicaque reportó el mayor número de registros (14) en la localidad de Camino a San Juan (CJ), de los 13 sitios en los que se observó y en el caso de Catasticta nimbice nimbice, el mayor registro (16) fue en Santa Catarina de los nueve en los que estuvo presente. 
Distribución altitudinal. Se consideró un gradiente altitudinal de los 1900 a los 3800 $\mathrm{m}$ que comprende el área de estudio (Cuadro 1), no obstante, la altitud mayor en la que se obtuvieron registros fue $3400 \mathrm{~m}$, que corresponde a la localidad de San Juan Tetla en el Estado de Puebla (Cuadro 1).

En este análisis las localidades fueron agrupadas según su ubicación en alguna de dos vertientes: en la occidental se incluyeron 14 sitios (La Tijera, Viveros, Tomacocos, Tetela, Camino a Hueyapan, Ocoxaltepec, Ejido Tlalmanalco, El Salto, San Rafael, Santa Catarina, Ávila Camacho, Llano Grande, Hueyapan y Ecatzingo) y 23 en la vertiente oriental (Buenavista, San Juan Tetla, Camino a San Juan, San Alejo, Tochimilco, San Nicolás, Tlatenco, Atzompa, Santa Rita, El Sifón, San Juan Cuauhtémoc, San Andrés, Atexcac, La Soledad, La Vaquería, Río Frío, Atlimeyaya, San Pedro Benito Juárez, Tianguismanalco, Santa Cruz, Los Venados, Valle Espinoso y Kilómetro 40) (Fig. 1).

Así, se encontró que de las 75 especies reconocidas, 58 fueron registradas en cada una de las vertientes. Sin embargo, es importante resaltar que 17 especies estuvieron presentes solo en una vertiente y que 41 de ellas se compartieron (Cuadro 4). De la misma manera, del total de registros de Papilionoidea (693), 309 ocurrieron en la occidental y 384 en la vertiente oriental. Además, en cada vertiente la riqueza y la abundancia fueron evaluadas a intervalos de $100 \mathrm{~m}$, definiendo un total de 16 pisos altitudinales en el gradiente en el que fueron observadas mariposas (1900 a $3400 \mathrm{~m}$ ), nueve para la vertiente occidental y 14 para la occidental (Cuadro 5). En cada piso se estandarizaron los valores de riqueza y abundancia, es decir, las especies fueron contabilizadas una sola vez y el número de registros dividido entre el número de localidades consideradas (RiqAjus y AbAjus) con el fin de realizar las comparaciones de manera más objetiva. Estos resultados se sintetizan en el Cuadro 5.

En este análisis se observó que la mayor concentración de especies ocurrió entre los 2400 y $2800 \mathrm{~m}$ en la vertiente occidental y en el intervalo de 2400 a $3100 \mathrm{~m}$ en la oriental. Además, los máximos de riqueza ocurrieron a distintos pisos altitudinales entre ambas vertientes, a $2400 \mathrm{~m}$ en la occidental ( 29 especies) y a $3000 \mathrm{~m}$ en la oriental (26 especies). El primero también representa el máximo de riqueza por piso altitudinal para toda el área (Fig. 6).

Con respecto a la abundancia, en este cuadro también se observa que el mayor número de registros ocurrió en los mismos intervalos altitudinales descritos para la riqueza ( 2400 y $2800 \mathrm{~m}$ en la vertiente occidental y 2400 a $3100 \mathrm{~m}$ en la oriental). Los valores máximos para ambas vertientes se presentaron en el piso de $2700 \mathrm{~m}$ con 51 registros en la oriental y 66 en la occidental; este último fue el valor máximo de abundancia para toda el área (Fig. 6).

Distribución por comunidad vegetacional. De acuerdo con la clasificación de los tipos de vegetación propuesta por INEGI (1988), en este trabajo se reconocieron once comunidades vegetacionales en la zona de estudio: bosque caducifolio (BCA), de oyamel (BY), Alnus (BAl), pino (BP), pino-encino (BPE), encino (BE), encino-pino $(B E P)$, mesófilo de montaña (BMM), ecotono entre selva baja caducifolia y bosque 
Luna-Reyes \& Llorente-Bousquets: Papilionoideos de la Sierra Nevada, México

mesófilo de montaña (SBC-BMM), zona perturbada, en este caso constituida principalmente por vegetación riparia (ZP), y cultivos (C), en las que se encuentran situadas las 37 localidades en las que se registraron papilionoideos (Cuadro 1).

\section{Cuadro 4}

Distribución de especies exclusivas por vertiente: OCC: occidental; OTE: oriental.

\begin{tabular}{|c|c|c|c|}
\hline FAMILIA & Especie & OCC & OTE \\
\hline \multirow[t]{3}{*}{ Papilionidae } & Papilio polyxenes asterius & & $\mathrm{E}$ \\
\hline & Parides photinus photinus & & $\mathrm{E}$ \\
\hline & Priamides pharnaces & & $\mathrm{E}$ \\
\hline \multirow[t]{7}{*}{ Pieridae } & Abaeis nicippe & $\mathrm{E}$ & \\
\hline & Colias eurytheme & & $\mathrm{E}$ \\
\hline & Pyrisitia nise nelphe & $\mathrm{E}$ & \\
\hline & Eurema boisduvaliana & & $\mathrm{E}$ \\
\hline & Hesperocharis costaricensis pasion & & $\mathrm{E}$ \\
\hline & Phoebis philea philea & $\mathrm{E}$ & \\
\hline & Pontia protodice & $\mathrm{E}$ & \\
\hline \multirow[t]{16}{*}{ Nymphalidae } & Anemeca ehrenbergii & $\mathrm{E}$ & \\
\hline & Anthanassa alexon alexon & $\mathrm{E}$ & \\
\hline & Cyclogramma bacchis & $\mathrm{E}$ & \\
\hline & Cyllopsis pyracmon pyracmon & & $\mathrm{E}$ \\
\hline & Chlosyne lacinia lacinia & $\mathrm{E}$ & \\
\hline & Dione juno huascuma & & $\mathrm{E}$ \\
\hline & Doxocopa laure acca & $\mathrm{E}$ & \\
\hline & Euptoieta hegesia hoffmanni & E & \\
\hline & Heliconius charitonia vazquezae & $\mathrm{E}$ & \\
\hline & Libytheana carinenta mexicana & $\mathrm{E}$ & \\
\hline & Pessonia polyphemus polyphemus & & E \\
\hline & Phyciodes pictus pallescens & $\mathrm{E}$ & \\
\hline & Polygonia haroldii & & $\mathrm{E}$ \\
\hline & Siproeta stelenes biplagiata & $\mathrm{E}$ & \\
\hline & Texola elada elada & & $\mathrm{E}$ \\
\hline & Vanessa atalanta rubria & & $\mathrm{E}$ \\
\hline \multirow[t]{7}{*}{ Lycaenidae } & Arawacus jada & & $\mathrm{E}$ \\
\hline & Callephelis perditalis perditalis & $\mathrm{E}$ & \\
\hline & Callophrys goodsonii & $\mathrm{E}$ & \\
\hline & Callophrys spinetorum & & $\mathrm{E}$ \\
\hline & Emesis mandana & $\mathrm{E}$ & \\
\hline & Erora quaderna & & $\mathrm{E}$ \\
\hline & Hemiargus ceraunus zachaeina & & $\mathrm{E}$ \\
\hline
\end{tabular}

En general, la mayor riqueza se encontró en el bosque de encino (Santa Catarina con 25 especies) y en el bosque mesófilo (Ecatzingo con 24 especies); en el primero ocurrió además la mayor abundancia (88 registros; Cuadro 1). De la misma manera que en el análisis altitudinal, la distribución de papilionoideos por comunidad fue evaluada por vertientes. En ambas se identificaron ocho comunidades vegetacionales: cinco de ellas fueron reconocidas en las dos vertientes (BE, BPE, BP, ZP y C) y tres más solo en alguna (BY, BCA y BMM en la occidental y BAI, BEP y SBC-BMM en la oriental; Cuadro 6). 

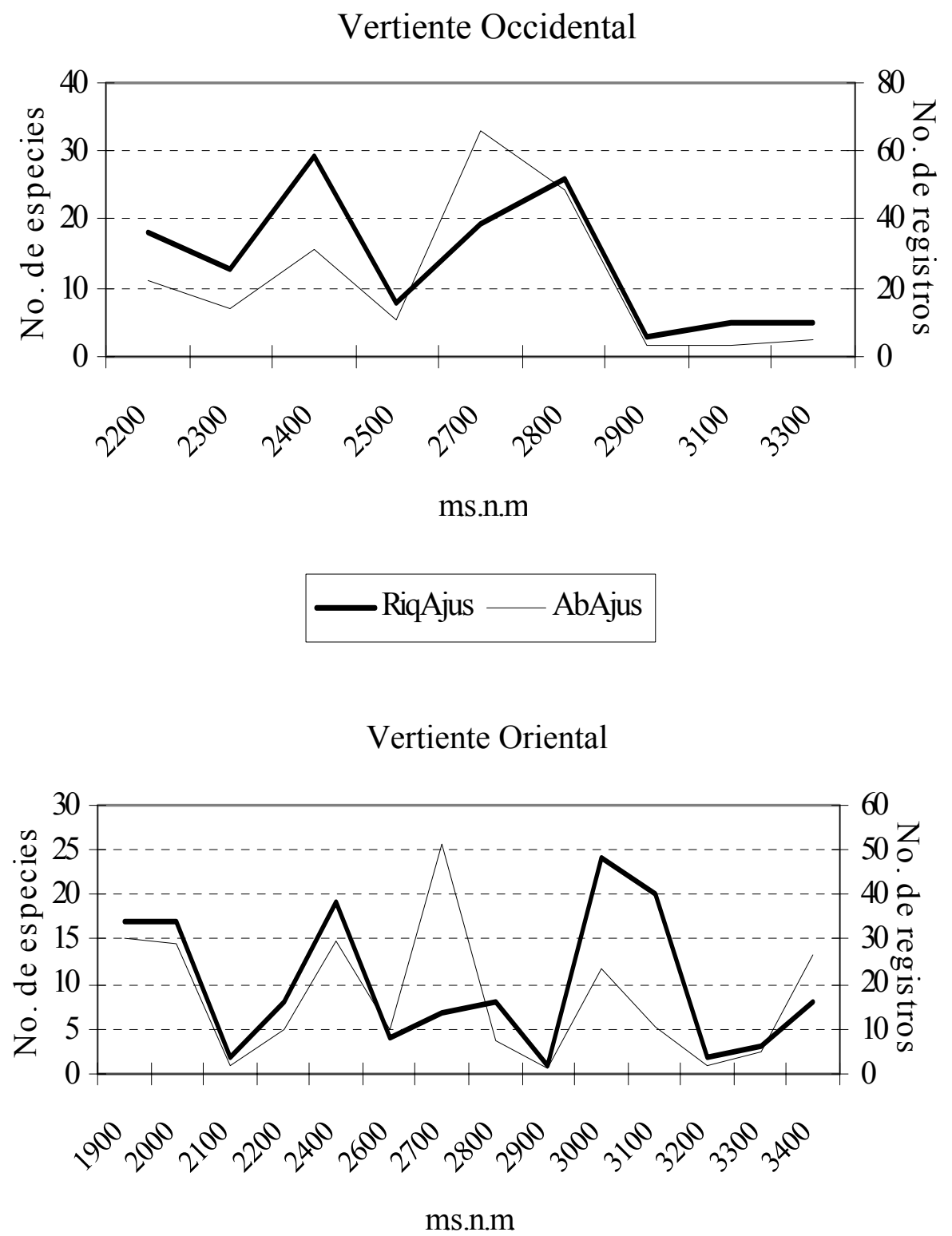

$$
\text { RiqAjus _ AbAjus }
$$

Figura 6

Distribución altitudinal de Papilionoidea. RiqAjus: riqueza ajustada; AbAjus: abundancia ajustada. 
Luna-Reyes \& Llorente-Bousquets: Papilionoideos de la Sierra Nevada, México

\section{Cuadro 5}

Distribución de Papilionoidea por piso altitudinal

\begin{tabular}{|c|c|c|c|c|}
\hline $\begin{array}{l}\text { Piso altitudinal } \\
\text { msnm }\end{array}$ & Vertiente & Localidades & RiqAjus & AbAjus \\
\hline 1900 & OTE & AJ & 17 & 30 \\
\hline 2000 & OTE & $\mathrm{TH}$ & 17 & 29 \\
\hline 2100 & OTE & TG & 2 & 2 \\
\hline 2200 & OCC & $\mathrm{TT}, \mathrm{CH}$ & 18 & 22.5 \\
\hline 2200 & OTE & $A Y$ & 8 & 10 \\
\hline 2300 & $\mathrm{OCC}$ & $\mathrm{HY}$ & 13 & 14 \\
\hline 2400 & $\mathrm{OCC}$ & $\mathrm{OX}, \mathrm{EC}$ & 29 & 30.5 \\
\hline 2400 & OTE & BJ, CZ & 19 & 29.5 \\
\hline 2500 & $\mathrm{OCC}$ & TM & 8 & 11 \\
\hline 2600 & OTE & AN & 4 & 10 \\
\hline 2700 & $\mathrm{OCC}$ & SL & 19 & 66 \\
\hline 2700 & OTE & $A Z$ & 7 & 51 \\
\hline 2800 & $\mathrm{OCC}$ & $A C, S C$ & 26 & 47.5 \\
\hline 2800 & OTE & CJ, CU, VQ & 8 & 7.66 \\
\hline 2900 & OCC & VV & 3 & 3 \\
\hline 2900 & OTE & RT & 1 & 1 \\
\hline 3000 & OTE & TN, SF, SO, VE & 26 & 23.25 \\
\hline 3100 & $\mathrm{OCC}$ & TJ, TL, SR & 5 & 4.5 \\
\hline 3100 & OTE & $\mathrm{AX}, \mathrm{NC}, \mathrm{RF}, \mathrm{LV}$ & 20 & 10.75 \\
\hline 3200 & OTE & $\mathrm{KM}$ & 2 & 2 \\
\hline 3300 & OCC & LL & 5 & 5 \\
\hline 3300 & OTE & BV & 3 & 5 \\
\hline 3400 & OTE & $\mathrm{JT}$ & 8 & 26 \\
\hline
\end{tabular}

Las claves de las localidades se describen en el cuadro 1. OCC: occidental; OTE: oriental; RiqAjus: riqueza ajustada; AbAjus: abundancia ajustada

La riqueza y la abundancia también fueron estandarizadas (RiqAjus y AbAjus, respectivamente): en cada comunidad se consideraron las especies acumuladas presentes en cada grupo de localidades, así como los valores promedio de abundancia, es decir, el número de registros dividido entre el número de sitios incluidos (Cuadro 6). De acuerdo con estos resultados, en el bosque de pino-encino de la vertiente oriental se registró el valor máximo de riqueza (30 especies) y en el bosque mesófilo de la vertiente occidental el mayor número de registros (55).

En el Cuadro 6 se observa que en la vertiente occidental el bosque caducifolio y el bosque de encino tuvieron la mayor riqueza ( 26 especies), y que el bosque de pino estuvo pobremente representado (una especie); en lo que se refiere a la abundancia, los valores máximos se encontraron en el bosque mesófilo y los mínimos en los bosques de oyamel y de pino. Por el contrario, en la vertiente oriental la comunidad más rica (30 especies) fue la de pino-encino y la más pobre (dos especies) representada por la vegetación perturbada; sin embargo, el encinar fue la comunidad vegetacional más abundante en mariposas y la vegetación perturbada la que tuvo el menor número de registros. Esta información se ilustra de manera sintética en la figura 7. 
Cuadro 6

Distribución vegetacional de Papilionoidea

\begin{tabular}{ccccc}
\hline Vertiente Hábitat & Localidades & $\begin{array}{c}\text { Altitud } \\
\text { msnm }\end{array}$ & RiqAjus & AbAjus \\
\hline Occidental & CH & 2200 & 2 & 11 \\
C & TT, TJ, TL, LL & $2200-3300$ & 26 & 11.5 \\
BCA & HY & 2300 & 13 & 14 \\
ZP & EC & 2400 & 24 & 55 \\
BMM & OX, TM, SL & $2400-2700$ & 24 & 27.66 \\
BPE & AC, SC & 2800 & 26 & 47.5 \\
BE & VV & 2900 & 3 & 3 \\
BY & SR & 3100 & 1 & 3 \\
BP & & & & \\
Oriental & AJ & 1900 & 17 & 30 \\
C & TH & 2000 & 17 & 29 \\
SBC-BMM & TG & 2100 & 2 & 2 \\
ZP & AZ, CZ & $2400-2700$ & 22 & 45 \\
BE & BJ, AN, CJ, RT, AX, NC, RF, JT & $2400-3400$ & 30 & 13.66 \\
BPE & CU, VQ, TN, SO, VE, LV & $2800-3100$ & 20 & 9 \\
BP & SF, KM & $3000-3200$ & 16 & 28.5 \\
BEP & BV & 3300 & 3 & 5 \\
BAl &
\end{tabular}

Las claves de vegetación y localidades se describen en el cuadro 1. RiqAjus: riqueza ajustada; AbAjus: abundancia ajustada.

\section{DISCUSIÓN}

Lista de especies. De las 75 especies reconocidas, 34 de ellas (45\%), en su mayoría ninfálidos y licénidos, son citadas en este trabajo por primera vez para el área. Las demás fueron registradas con anterioridad para una zona de bosque mesófilo de montaña en la localidad de San Rafael, Estado de México (Barrera \& Romero 1986) y otras en la base de datos de mariposas de CONABIO (2001) para siete localidades de la Sierra Nevada. Las especies Pyrisitia proterpia, Myscelia ethusa, Anaea troglodyta, Hypanartia lethe y Chorostrymon simaethis fueron citadas por estas autoras y Calaides androgeus, Pyrrhosticta victorinus victorinus, Heraclides thoas, Parides montezuma y Eucheira socialis, además de Pyrisitia proterpia fueron registradas por CONABIO. Excepto la última (capturada posteriormente en un sitio que no se incluye en este trabajo), ninguna de estas especies fue observada en el presente estudio.

$\mathrm{La}$ adición de estas especies citadas con anterioridad, hace un total de 85 reconocidas hasta la fecha para la zona y constituye el primer inventario de Papilionoidea para la Sierra Nevada.

Esfuerzo de captura. De acuerdo con el indicador empleado $\left(\mathrm{ChaO}_{2}\right)$, la relación de especies que se ofrece en este trabajo (75) representa el $82 \%$ del total estimado para el área. 
Luna-Reyes \& Llorente-Bousquets: Papilionoideos de la Sierra Nevada, México

Vertiente Occidental

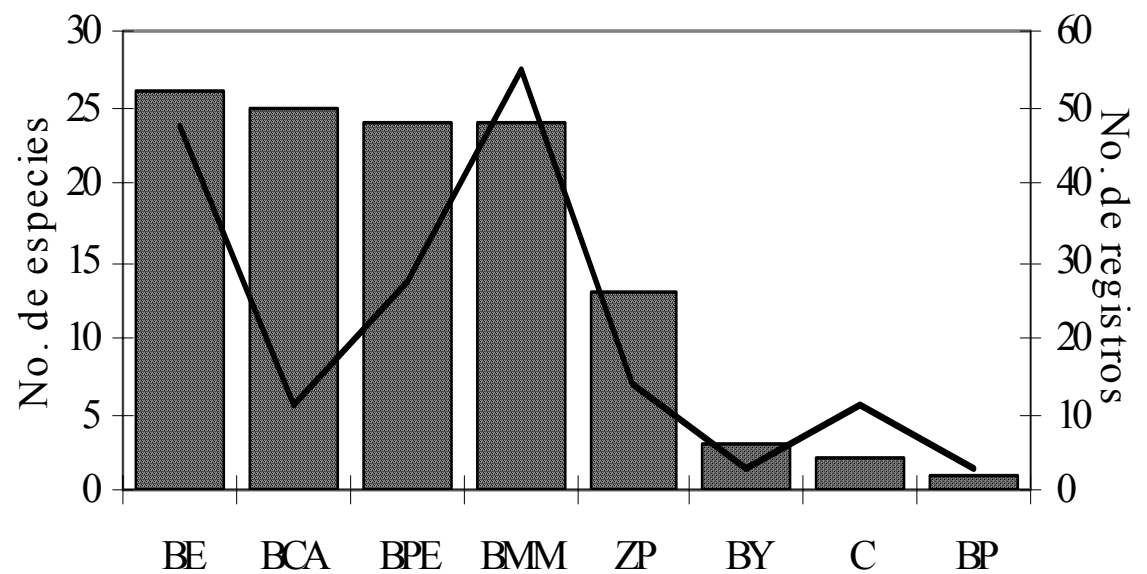

RiqAjus —AbAjus

Vertiente Oriental

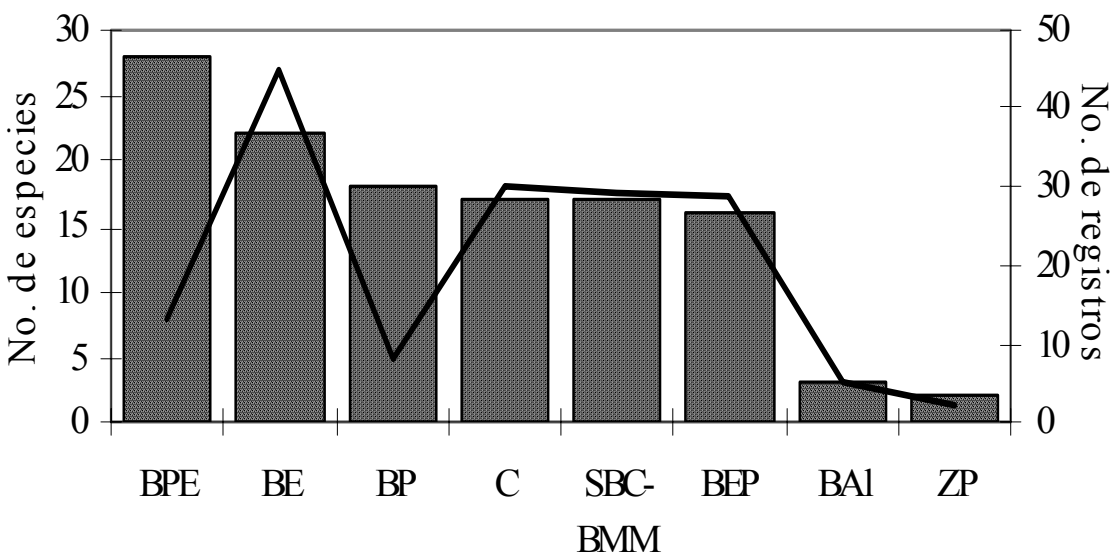

RiqAjus -AbAjus

Figura 7

Distribución de Papilionoidea por tipo vegetacional. Las siglas corresponden a las del Cuadro 1. RiqAjus: riqueza ajustada; AbAjus: abundancia ajustada. 
Riqueza. El número de especies encontrado en la Sierra Nevada es mayor al obtenido en Nanchititla y San Rafael en el Estado de México, Los Dínamos, D. F., y en El Chico, Hidalgo (Cuadro 2). La diferencia puede explicarse en función de la mayor amplitud del gradiente altitudinal considerado en este estudio, así como el mayor número de comunidades vegetacionales comprendidas, lo que ofrece mayor cantidad de microhábitats y por consiguiente, mayor número de especies de flora que son utilizadas por las distintas especies de papilionoideos; en síntesis es mayor heterogeneidad ambiental. Por otro lado, también es importante considerar que el esfuerzo de captura (número de horas de colecta multiplicado por el número de recolectores) ejercido en la Sierra Nevada (544 horas) fue mayor al efectuado en Nanchititla (12 días; no se tiene el dato del número de horas por día), pero comparativamente menor al de San Rafael (572 horas), El Chico (877 horas) y Los Dínamos (1248 horas de trabajo efectivo).

La proporción de cada familia de Papilionoidea registrada en el área de estudio confirma el comportamiento detectado en las cuatro localidades mencionadas, en las que Nymphalidae fue la mejor representada pues le correspondió el mayor número de especies, seguida de Pieridae, Lycaenidae y Papilionidae, en ese orden (Fig. 2).

Abundancia. El número de registros de papilionoideos en la Sierra Nevada fue considerablemente más bajo (menos de la mitad) que el observado en Los Dínamos, San Rafael y El Chico (Cuadro 2). En estos trabajos las colectas se efectuaron cada semana durante varios meses de manera sistemática en un número menor de localidades (nueve, seis y tres, respectivamente), mientras en la zona de estudio las visitas a cada sitio fueron puntuales. Aunado a esto, es necesario considerar que en varios sitios visitados en este trabajo se presentaron condiciones atmosféricas desfavorables para la colecta de mariposas (bajas temperaturas, neblina, lluvia, etc.), lo cual es un factor de explicación relevante pues las mariposas son poikilotermas y acentuadamente fotofílicas.

En cuanto a la distribución de registros por familia, se observó un patrón coincidente al citado en San Rafael y en El Chico, en el que Pieridae fue la más abundante (42\%), después Nymphalidae (31\%), Lycaenidae (19\%) y Papilionidae (8\%), pero distinto al encontrado en Los Dínamos, en donde los ninfálidos fueron los más numerosos. En esta última localidad, el $60 \%$ de los registros de Nymphalidae correspondieron solo a la especie Paramacera xicaque xicaque (1254 ejemplares), lo cual incrementó de manera importante la proporción de esta familia en el área.

Por otro lado, se reconoció la tendencia general de la abundancia en la que pocas especies fueron abundantes y la mayoría escasas; las más abundantes en el área de estudio fueron Catasticta teutila teutila (79 registros), C. nimbice nimbice (47), Paramacera xicaque xicaque (49) y Leptophobia aripa elodia (53). Estas cuatro especies estuvieron presentes también entre las más numerosas en las localidades de San Rafael, Los Dínamos y El Chico, aunque con una jerarquía de abundancia distinta.

Distribución por localidad. Santa Catarina (SC) fue la localidad con mayor riqueza y abundancia de todo el estudio (Cuadro 1); sin embargo, también destacan las 
localidades de Ecatzingo (EC) y El Salto (SL), todas ellas ubicadas en el Estado de México. Por el contrario, las localidades de Santa Rita (RT) y Cuauhtémoc (CU), fueron las más pobres en cuanto al número de especies y de registros de papilionoideos.

La mayor parte de las especies $(70 \%)$ tuvo una distribución restringida, con una proporción alta de especies presentes solo en una localidad. Leptophobia aripa elodia fue la especie de mayor distribución en la Sierra Nevada, además de ser una de las especies más abundantes. Las otras tres especies con mayor abundancia dentro del área (Catasticta teutila teutila, C. nimbice nimbice y Paramacera xicaque xicaque) tuvieron sus poblaciones mayores en sitios determinados (uno a tres), entre los que destaca Santa Catarina. El patrón de distribución de estas cuatro especies corresponde al citado para San Rafael, Los Dínamos y El Chico.

Distribución altitudinal. En cuanto a la disposición altitudinal de las especies de Papilionoidea en el área de estudio, se encontró que $3400 \mathrm{~m}$ fue la cota altitudinal máxima de su distribución, es decir, en altitudes mayores no se observaron mariposas. En este sentido, es posible que el clima y, en particular, la temperatura jueguen un papel determinante en la distribución de papilionoideos. Arriaga et al. (2000) definieron tres subtipos climáticos templados para la cota superior a los $2800 \mathrm{~m}$ en la Sierra Nevada: $\mathrm{Cb}^{\prime}(\mathrm{w} 2)$ y $\mathrm{C}(\mathrm{w} 2)$ y $\mathrm{C}(\mathrm{w} 2) \mathrm{x}^{\prime}$; que entre ellos se distinguen principalmente por sus valores de temperatura y en menor proporción por la precipitación; el primer subtipo está presente en la mayor parte de la Sierra Nevada y su temperatura media anual oscila entre $5^{\circ} \mathrm{C}$ y $12^{\circ} \mathrm{C}$, a diferencia de los dos últimos en los que la variación de la temperatura media anual se presenta entre $12^{\circ} \mathrm{C}$ y $18^{\circ} \mathrm{C}$. Aunque no se tienen estos datos para las localidades visitadas, durante el trabajo de campo se constató que no se observaron papilionoideos en los sitios más altos y, como consecuencia, los más fríos.

En este análisis, las localidades fueron agrupadas por vertiente y luego por piso altitudinal de acuerdo con su ubicación. De esta manera se encontró que la riqueza fue la misma en ambas vertientes (58 especies) y que la abundancia fue mayor en la vertiente oriental (384 registros); en este aspecto es necesario considerar que, a pesar que en la vertiente oriental estuvieron ubicados un mayor número de sitios (23), las localidades en las que detectó la mayor riqueza y abundancia se ubicaron precisamente en la occidental (SC, EC, SL y TT). Por otro lado, alrededor de la mitad $(44 \%)$ de las especies fueron exclusivas para una u otra vertiente, lo cual sugiere que la composición lepidopterofaunística entre ambas es distinta (Cuadro 5). Por ejemplo, la presencia de las especies Enantia mazai diazi, Cyclogramma bacchis y Doxocopa laure aca indica que la vertiente occidental tiene gran influencia de la Depresión del Balsas.

Además, se reconoció el patrón general en el que el número de especies se reduce conforme aumenta la altitud, de manera más clara en la vertiente occidental (Fig. 6). Esta tendencia también fue registrada en la Sierra de Juárez (Luis et al. 1991) y en la Sierra de Manantlán (Vargas et al. 1999) donde se consideraron intervalos altitudinales similares al que aquí nos ocupa. Este patrón también es coincidente al encontrado por Monteagudo et al. (2001) en un análisis altitudinal más amplio (300 a 2450 m) en el que fueron consideradas diversas localidades en transectos de la Sierra Madre del Sur. 
En el piso de los $2400 \mathrm{~m}$ de la vertiente occidental se detectaron los valores máximos de riqueza ( 29 especies) y en el de $2700 \mathrm{~m}$ los máximos de abundancia para todo el estudio (Cuadro 5). En general, pudo observarse que la distribución de las especies y sus poblaciones, así como los valores máximos, se presentaron en distintos pisos altitudinales entre ambas vertientes, ocurriendo en puntos más altos y a intervalos más amplios en la vertiente oriental. Como se comprobó durante el desarrollo del trabajo de campo, la temperatura es más cálida en la parte oriental de la Sierra Nevada, a diferencia de la vertiente occidental donde además de presentarse temperaturas más bajas, también existe una mayor humedad, demostrada por la mayor incidencia de nublados, lloviznas y presencia de neblina. Este hecho confirma la importancia que tiene la temperatura con respecto a la disposición altitudinal de las mariposas en la Sierra Nevada.

Distribución por comunidad vegetacional. Entre las 11 comunidades vegetacionales reconocidas en la Sierra Nevada, los bosques de encino y mesófilo de montaña tuvieron los valores máximos de riqueza de todo el estudio; estas comunidades vegetacionales también fueron citadas como las más ricas en Los Dínamos, D.F. (Luis \& Llorente 1990).

En el análisis general de la distribución de Papilionoidea por comunidad vegetacional, la vertiente oriental mostró la mayor riqueza (bosque de pino-encino con 30 especies) y la occidental la mayor abundancia (bosque mesófilo de montaña con 55 registros). También es importante mencionar que no hubo correspondencia entre riqueza y abundancia de las cinco comunidades compartidas por ambas vertientes. Por ejemplo, los bosques de pino, pino-encino y los cultivos tuvieron mayor número de especies y de registros en la vertiente oriental, mientras las comunidades de bosque de encino y la de vegetación perturbada fueron más ricas y abundantes en la vertiente occidental. Durante el trabajo de campo se observó que, en general, la vegetación de la vertiente oriental está muy perturbada y que en muchos sitios las comunidades vegetacionales naturales alternan con zonas de cultivos (manzana, pera, durazno, cebada, trigo, principalmente) propiciando áreas abiertas con gran cantidad de plantas en floración que resultan muy productivas en lo que se refiere a la captura de papilionoideos. Por el contrario, la vertiente occidental está mejor conservada, a pesar de la explotación forestal frecuente, tal vez debido al estricto control que se ejerce con respecto al acceso a la zona. Es probable, además, que estas diferencias se deban a que las comunidades vegetacionales que tuvieron la mayor riqueza y abundancia incluyeron sitios que en su conjunto ocuparon un intervalo altitudinal mayor (Cuadro 6). En el caso de los cultivos, los valores más altos se presentaron a menor altitud (1900 $\mathrm{m}$ ) en el sitio denominado San Alejo (AJ); ésta es una área abierta, sin elevaciones (la de altitud menor en todo el estudio) en la que se observaron cultivos de frijol y calabaza y gran cantidad de plantas herbáceas en floración. Como se sabe, el néctar de las flores es una de las fuentes alimenticias importantes para el gremio de nectarívoras en una comunidad de mariposas adultas (Vargas et al. 1994), por lo que estos insectos son atraídos hacia los lugares donde abundan las inflorescencias. 
Luna-Reyes \& Llorente-Bousquets: Papilionoideos de la Sierra Nevada, México

La vegetación presente en Tochimilco fue reconocida como una comunidad transicional entre selva baja caducifolia y bosque mesófilo de montaña (SBC-BMM). Aunque la vegetación presente en las laderas circundantes a esta localidad es selva baja caducifolia, la fisonomía del sitio de colecta es distinta. En esta localidad se capturó el piérido Enantia mazai diazi, especie que ha sido citada en Nayarit, Colima, Jalisco, Guerrero y Oaxaca en localidades de bosques mesófilo y tropical subcaducifolio y en las selvas alta o mediana perennifolia o subperennifolia y baja caducifolia (Llorente 1984, De la Maza 1987, Luis et al. 1991, Vargas et al. 1994, 1999), por lo que es posible que esta comunidad vegetacional se trate de una zona de ecotono entre selva baja caducifolia y bosque mesófilo de montaña como aquí se propone.

\section{AGRADECIMIENTOS}

Esta investigación se desarrolló gracias al apoyo de la Carrera de Biología de la Facultad de Estudios Superiores Zaragoza, UNAM. El segundo autor agradece al proyecto DGAPA IN-218502 y CONACYT 36488. Reconocemos el apoyo de Armando Luis Martínez en la confirmación o determinación taxonómica de algunos ejemplares. Especialmente a Carolina Poveda Matallana y Camilo Rojas Parra por su valiosa participación en el diseño y elaboración del mapa y al alumno Alejandro Mora Contreras por su entusiasta colaboración en la colecta y procesamiento del material entomológico. A dos de los revisores anónimos, cuyas observaciones y sugerencias mejoraron el presente trabajo.

\section{LITERATURA CITADA}

Arita, H. \& L. León P. 1993. Diversidad de mamíferos terrestres. Ciencias. No. Especial, 7: $13-22$.

Arriaga, L., J.M. Espinoza, C. Aguilar, E. Martínez \& L. Gómez. 2000. Regiones terrestres prioritarias de México. CONABIO. México.

Barrera, A. \& M. E. Díaz B. 1977. Distribución de algunos lepidópteros de la Sierra de Nanchititla, México, con especial referencia a Tisiphone maculata Hpff. (Ins.: Lepid.). Rev. Soc. Mex. Lep., 3(1): 17-28.

Barrera, G. T. \& L. Romero H. 1986. Estudio faunístico de lepidópteros (Superfamilia Papilionoidea) en un bosque mesófilo de montaña en Cascada de Los Diamantes, San Rafael, Estado de México. Tesis de Licenciatura. Escuela Nacional de Estudios Profesionales Zaragoza. UNAM. México. $58 \mathrm{p}$.

Bizuet, F. Y., A. Luis M. \& J. Llorente B. 2001. Mariposas del Parque Nacional El Chico, Hidalgo y sus relaciones biogeográficas con cinco zonas aledañas al Valle de México, México (Lepidoptera: Papilionoidea). SHILAP Revta. Lepid., 29(114): 145-159.

Colwell, R. K. \& J.A. Coddington. 1994. Estimating terrestrial biodiversity through extrapolation. Phil. Trans. Roy. Soc. London Series B., 345: 101-118.

CONABIO. 2001. Lepidópteros (Papilionoidea) de la Región Prioritaria Terrestre 107 (Sierra Nevada). Reporte de la consulta a la base de datos de mariposas. (Claudia Aguilar).

De la Maza, R. R. 1987. Mariposas mexicanas. Fondo de Cultura Económica. México. 302 p.

DeVries, J. P. 1987. The butterflies of Costa Rica and their natural history. Papilionidae, Pieridae, Nymphalidae. Princeton University Press. New Jersey. 327p.

Ehrlich, P.R. \& A.H. Ehrlich. 1961. How to know the butterflies. WM. C. Brown Company Pub. Dubuque, lowa. 262 p.

Emmel, T.C. 1975. Butterflies. Chanticleer Press. New York. 260 p. 
Flores, V. O. 1993. Riqueza de anfibios y reptiles. Ciencias. No. Especial, 7: 33-42.

Flores, V. O. \& P. Gerez. 1994. Biodiversidad y conservación en México: vertebrados, vegetación y uso del suelo. UNAM-CONABIO. México. 439 p.

Heppner, J. B. 1991. Faunal regions and the diversity of Lepidoptera. Trop. Lepid., 2 (Suppl. 1): $1-85$. 1): $1-148$.

Howe, W. H. 1975. The butterflies of North America. Doubleday \& Company, Inc. New York. 633 p.

INEGI. 1988. Carta de uso del suelo y vegetación. Secretaría de Programación y Presupuesto. México. Esc. 1: 1000000.

Kristensen, N. P. 1975. Remarks on the family-level phylogeny of butterflies (Insecta: Lepidoptera, Rhopalocera). Zool. Syst. Evol. Forsch., 14: 23-33.

Llorente, B. J. 1984. Sinopsis sistemática y biogeográfica de los Dismorphiinae de México, con especial referencia al género Enantia Huebner (Lepidoptera: Pieridae). Folia Entomol. Mex. 58: 1-207.

Llorente, B. J., A. Luis; I. Vargas F. \& J. Soberón M. 1996. Papilionoidea (Lepidoptera), pp. 531-348. In: Llorente, J.; A. García y E. González (comps.): Biodiversidad, taxonomía y biogeografía de artrópodos de México: hacia una síntesis de su conocimiento. CONABIO-UNAM.

Llorente, B. J., L. Oñate, A. Luis M. \& I. Vargas F. 1997. Papilionidae y Pieridae de México: Distribución geográfica e Ilustración. UNAM. México. 227 p.

Luis, M. A. \& J. Llorente B. 1990. Mariposas del Valle de México: Introducción e Historia I. Distribución local y estacional de los papilionoidea de la Cañada de los Dínamos; Magdalena Contreras, D. F., México. Folia Entomol. Mex. 78. 95-198.

Luis, M. A., J. Llorente B., I. Vargas F. \& A. L. Gutiérrez. 2000. Síntesis preliminar del conocimiento de los Papilionoidea (Lepidoptera: Insecta) de México. Pp. 275-285. In: Martín P. F., J. J. Morrone y A. Melic. (Eds.) Monografías Tercer Milenio. Vol. I. Boletín SEA. Zaragoza, España.

Luis, M. A., J. Llorente B., I. Vargas F. \& A. D. Warren. 2003. Biodiversity and biogeography of Mexican butterflies (Lepidoptera: Papilionoidea and Hesperoidea). Proc. Entomol. Soc. Wash. 105(1): 209-224.

Luis, M. A., I. Vargas F. \& J. Llorente B. 1991. Lepidopterofauna de Oaxaca I: Distribución y fenología de los Papilionoidea de la Sierra de Juárez. Publ. Esp. Mus. Zool. Fac. Ciencias, UNAM, No. 3118 p.

Monteagudo, S. D., A. Luis M., I. Vargas F. \& J. Llorente B. 2001. Patrones altitudinales de diversidad de mariposas en la Sierra Madre del Sur (México). (Lepidoptera: Papilionoidea). SHILAP Revta. lepid. 29(115): 207-237.

Navarro, A. \& H. Benítez. 1993. Patrones de riqueza y endemismo de las aves. Ciencias. No. Especial, 7: 45-54.

Pyle, R. M. \& A. A. Knopf. 1981. The Audubon Society field guide to North American butterflies. Chanticleer Press. New York. 916 p.

Scott, J. A. 1985. The phylogeny butterfly (Papilionidae and Hesperidae). J. Res. Lepid., 23(4): 241-281.

. 1986. The butterflies of North America. A natural history and field guide. Stanford Univ. Press. California. 583 p.

Smart, P. 1989. The illustrated encyclopedia of the butterfly world. Crescent Books. New York. 275 p. 
Luna-Reyes \& Llorente-Bousquets: Papilionoideos de la Sierra Nevada, México

Vargas F. I., J. Llorente B. \& A. Luis M. 1994. Listado lepidopterofaunístico de la Sierra de Atoyac de Álvarez en el Estado de Guerrero: notas acerca de su distribución local y estacional (Rhopalocera: Papilionoidea). Folia Entomol. Mex. 86: 41-178. . 1999. Distribución de los Papilionoidea (Lepidoptera: Rhopalocera) de la Sierra de Manantlán (250-1650 m) en los Estados de Jalisco y Colima. UNAM. Publ. Esp. Mus. Zool. Fac. Ciencias, UNAM, 11: 1-153.

Recibido: 2 de junio 2003

Aceptado: 2 de febrero 2004 